\title{
Percepção na Utilização das Tecnologias de Informação e Comunicação em Quatro Escolas do Ensino Médio do Recife, PE
}

\author{
Ana Luiza Rodrigues ${ }^{1}$, Vladimir Homobono Soares ${ }^{1,2}$ \\ ${ }^{1}$ Escola Politécnica de Pernambuco - Universidade de Pernambuco \\ R. Benfica, 455 - Madalena, Recife - PE, 50720-001 - Brasil \\ ${ }^{2}$ Programa de Pós-Graduação em Engenharia Elétrica \\ Departamento de Eletrônica e Sistemas - Universidade Federal de Pernambuco \\ Av. Prof. Moraes Rego, 1235 - Cidade Universitária, Recife - PE, 50670-901 - Brasil \\ vladimirapoli.br
}

\begin{abstract}
This article seeks to investigate the degree of adhesion to the Information and Communication Technologies (ICT) by helping the students to use the resources more appropriately in schools to facilitate learning. A quest with high school students from four state public schools in the city of Recife, Pernambuco, Brazil, was carried out to investigate whether the teaching methodologies adopted ICTs and if they are being used effectively. We also analyzed the students' perceptions regarding the concept of "science" and "ICTs".
\end{abstract}

Resumo. Este artigo procura investigar o grau de adesão às Tecnologias de Informação e Comunicação (TIC), ajudando os alunos a usar os recursos de forma mais adequada nas escolas para facilitar a aprendizagem. Uma pesquisa com alunos do ensino médio de quatro escolas públicas estaduais da cidade do Recife, Pernambuco, foi realizada para investigar se as metodologias de ensino adotadas agregam as TICs, e se estas estão sendo utilizadas de maneira eficaz. Também foram analisadas as percepções dos alunos a repeito do conceito de "ciência" e de "TICs".

\section{Introdução}

Inovações tecnológicas que deixam a sociedade em um estado de contínua evolução são cada vez mais frequentes. Essas inovações são responsáveis por transformações no meio ambiente, na política, na economia, na sociedade e na educação. As ferramentas desenvolvidas nesse processo como, as redes de computadores, também causaram uma revolução na forma, rapidez e facilidade com que as informações são trocadas, difundidas e manipuladas nas diversas áreas do conhecimento humano, possibilitando significativas mudanças no tradicional ensino de ciência e tecnologia ofertado pelas escolas (Campanário, 2002; Anjos et al, 2014).

As escolas são incumbidas de desenvolver no aluno um senso crítico embasado na compreensão científica e tecnológica da realidade social e política na qual vive para que possa, desde cedo, aprender a tomar decisões responsáveis sobre questões de ciência e tecnologia na sociedade e atuar na solução de tais questões. Nesse contexto, uma das 
áreas pretendidas pelas TICs é de auxiliar o ensino e aprendizagem fornecendo recursos para troca de informação (Melnikoff, 2010; Santos, 2007).

Com o desenvolvimento de novas TICs, novos modelos de ensino e aprendizagem permitirão a expansão da criatividade, a aproximação e troca de experiências entre instituições, professores e alunos que antes eram limitados pela geografia, promovendo cursos de qualidade a distância, a dinamização dos conteúdos, a globalização das informações. Com a ampliação da Internet no mundo, a informação é disseminada de maneira rápida, ficando disponível pouco tempo após um acontecimento. Barvinski e Odakura (2005) afirmam que "Em um mundo permeado pela TI, em que as atividades profissionais tendem a imaterialidade, naturalmente não há como o Ensino manter-se alheio as TICs incorporando-as em seu dia a dia" ressaltando a importância da utilização das TICs no ensino.

Com a velocidade em que a informação é propagada, é possível se deparar com conceitos e dados incorretos. Nesta situação, é necessário, também, avaliar e refletir para que um conjunto de informações incorretas não formem falsos conhecimentos. A melhor forma de rejeitar ou validar uma informação é aplicar, por exemplo, um teste utilizando o método científico (Wernek, 2006; Montanari e Pilatti, 2011; Lakatos, 2003).

Ao longo do século XX vários conceitos foram formulados para definir o método cientifico. Entre as vertentes epistemológicas, há um tronco comum para tratar a elaboração de um conceito na ciência, como é dito por Massoni (2005), "a ciência é concebida construindo-se hipóteses (conjeturas) e confrontando-as, posteriormente, com o mundo real". Portanto, ao receber uma informação, é necessário conjecturar a respeito e confrontar com outras fontes de informação para, a partir daí, tentar concluir se tal fato é verdadeiro. Dessa forma, a utilização do método científico auxilia na formação do pensamento crítico, contribuindo positivamente para obtenção do conhecimento.

A tentativa de unificar as TICs com o método científico na prática pedagógica no ensino médio parte em conhecer as limitações e possíveis potencialidades existentes na rede pública de ensino para aplicar tal conceito. Neste contexto, foram investigados, por meio de um questionário semiestruturado, qual a percepção dos alunos a respeito de ciência e TICs, avaliando a disponibilidade dos dispositivos e o nível experiência dos alunos com equipamentos e aplicações mais comuns de acesso à Internet, a utilização de aplicações para estudos on-line e, por fim, se a escola possui estrutura para dar suporte as atividades utilizando TICs.

\section{Metodologia}

Este artigo está embasado em uma pesquisa que consiste em um questionário com dez perguntas respondidas por quarenta alunos. A distribuição dessa quantidade se deu entre as três séries do ensino médio de quatro escolas estaduais do Recife. $\mathrm{O}$ intuito foi verificar, de forma preliminar, a argúcia dos alunos no contexto das TICs utilizando análise de conteúdo.

A utilização da análise de conteúdo, descrita inicialmente por Bardin (2011), é muito útil para avaliar as respostas obtidas. A apreciação pode apresentar uma abordagem qualitativa, descrevendo de maneira objetiva e sistemática os atributos 
dentro de um contexto, ou uma descrição de maneira quantitativa, partindo das frequências em que características são escolhidas a partir de um questionário em uma população.

O intuito é organizar e ordenar elementos-chave do texto (palavras ou frases frequentes), para inferir uma expressão representativa dentro do contexto analisado. Essa metodologia busca relacionar termos semânticos (significantes) com estruturas sociológicas (significado), dividindo-se nas fases de pré-análise, codificação do material e tratamento dos resultados, suas inferências e interpretações (Bardin, 2011).

O questionário aborda, em perguntas objetivas, a frequência da utilização do computador (na escola, em casa ou lan house), a finalidade do acesso à Internet (busca por informações gerais ou pesquisas escolares), se os professores utilizam o computador para ministrar as aulas, se há laboratório de informática e/ou multimídia e quais recursos são utilizados nas aulas. As perguntas dissertativas questionam graus de interesse em disciplinas, o que poderia fazer uma aula mais interessante e se um recurso tecnológico contribui para o aprendizado. Além disso, é questionado qual o conceito a respeito de ciência, de tecnologia e de tecnologias de informação e comunicação.

\section{Resultados e Discussão}

Foram entrevistados alunos entre 14 e 18 anos, de ambos os sexos, envolvendo as Escola de Aplicação do Recife FCAP/UPE; Escola Técnica Estadual Cícero Dias; Escola Estadual Manoel Borba; Escola da Referência em Ensino Médio Santos Dumont.

Como pode ser visto na Figura 1a, dos alunos entrevistados, 95\% utilizam "frequentemente" ou "às vezes" o computador de maneira geral. De acordo com a Figura 1b, 87,5\% dos alunos relatam que há a utilização do computador pelos professores.

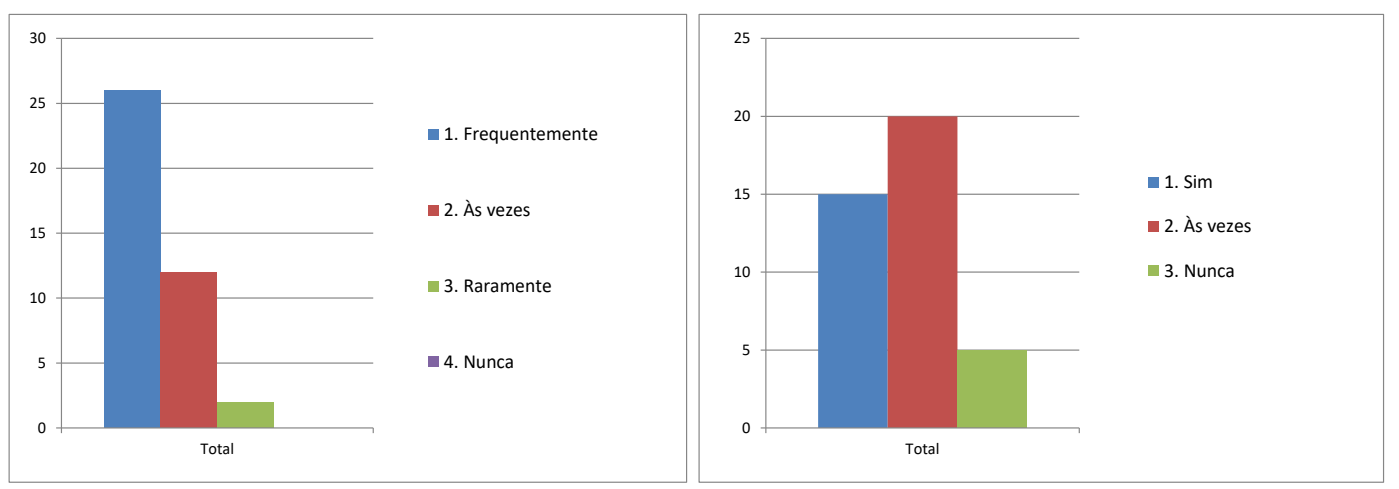

Figura 1: (a) Utilização do computador pelos alunos e se os (b) professores empregam seu uso em sala aula.

Com relação ao acesso à Internet, as maiores incidências são para "fins de comunicação (e-mail, skype e whatsapp)" e para "pesquisa de assuntos escolares" (Figura 2). Nessa questão, o aluno poderia assinalar mais de uma opção para o uso do Internet. De modo específico, apesar da incidência maior na utilização "às vezes" para o uso do computador na Escola Manoel Borba, foi seguido a tendência geral para uso do 
VI Congresso Brasileiro de Informática na Educação (CBIE 2017)

Anais do XXIII Workshop de Informática na Escola (WIE 2017)

acesso à Internet. Perceba que, para avaliar o acesso à Internet, foi possível escolher mais de uma opção.

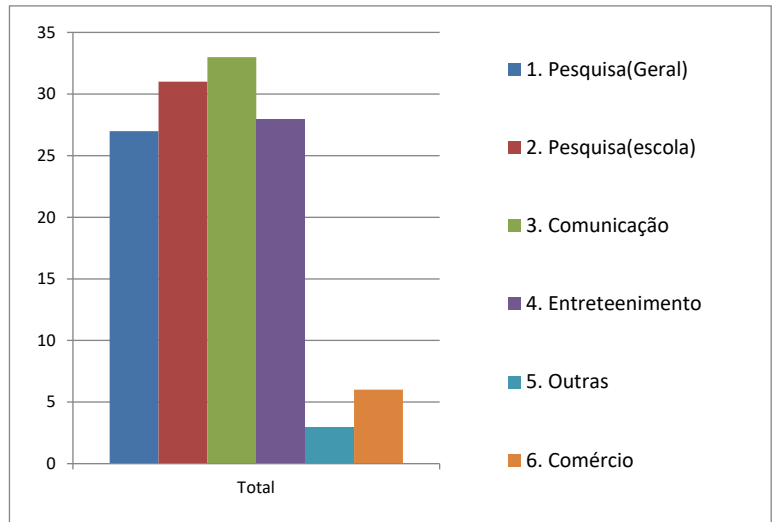

Figura 2: Incidência para as principais motivações para o acesso à Internet.

A respeito da percepção dos alunos no uso, por professores, de ferramentas que auxiliem o processo de aprendizagem, a Figura 3 apresenta os resultados obtidos na entrevista. É importante observar que, na visão dos alunos, "vídeo" apareceu como quarta opção mais utilizada (24 vezes) atrás de "quadro negro/branco" (40 vezes), "data show" (36 vezes) e, imediatamente após à utilização do "livro didático" (28 vezes). Associado às informações do uso, é exibido, na Figura 4, que, os principais recursos empregados para facilitar o aprendizado, segundo os alunos, os mais citados foram "vídeo" (28 vezes) e "data show" (27 vezes). Na Figura 5 estão as principais ideias descritas pelos alunos a respeito do uso desses recursos para o seu aprendizado. A maior incidência relaciona que o uso dos recursos torna a "praticidade para aprender $\mathrm{o}$ conteúdo" (16 vezes) mais viável.

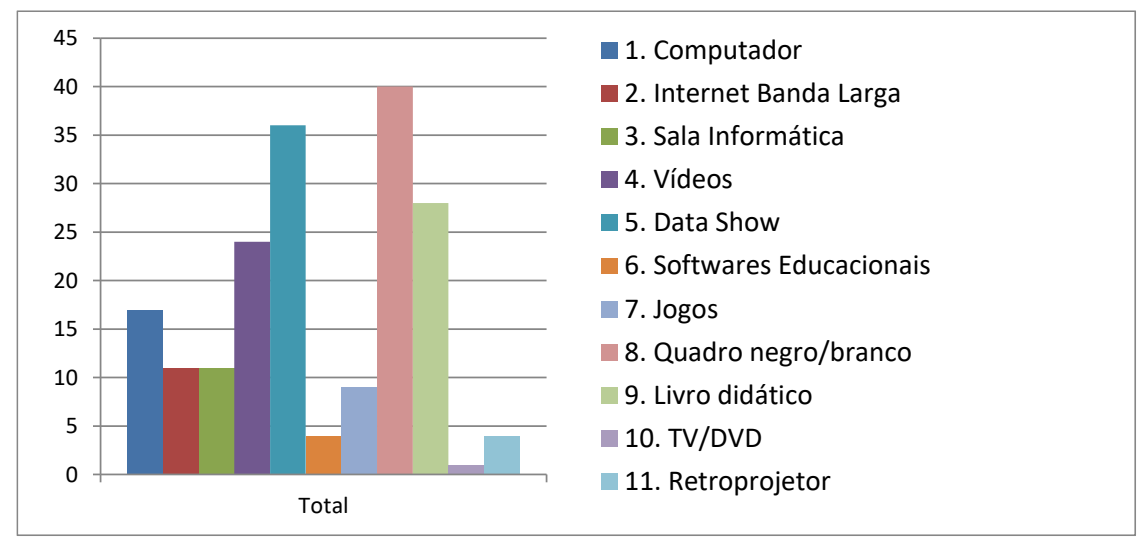

Figura 3: Percepção do aluno na utilização dos recursos em sala.

Como observado, há uma evidência de que existem alguns recursos disponíveis para o emprego da utilização do computador em sala de aula por professores por exemplo, porém, como é relatado por Lima e Castro (2016): 
VI Congresso Brasileiro de Informática na Educação (CBIE 2017)

Anais do XXIII Workshop de Informática na Escola (WIE 2017)

Apenas a presença de computadores em sala de aula não oferece contribuições relevantes aos processos de ensino e de aprendizagem. Há sempre o risco de serem utilizados dentro de uma perspectiva tradicional de educação, reforçando o modelo transmissor de informações, mantendo a postura passiva do aluno sem incentivá-lo a utilizar os instrumentos computacionais de forma colaborativa. (p.428)

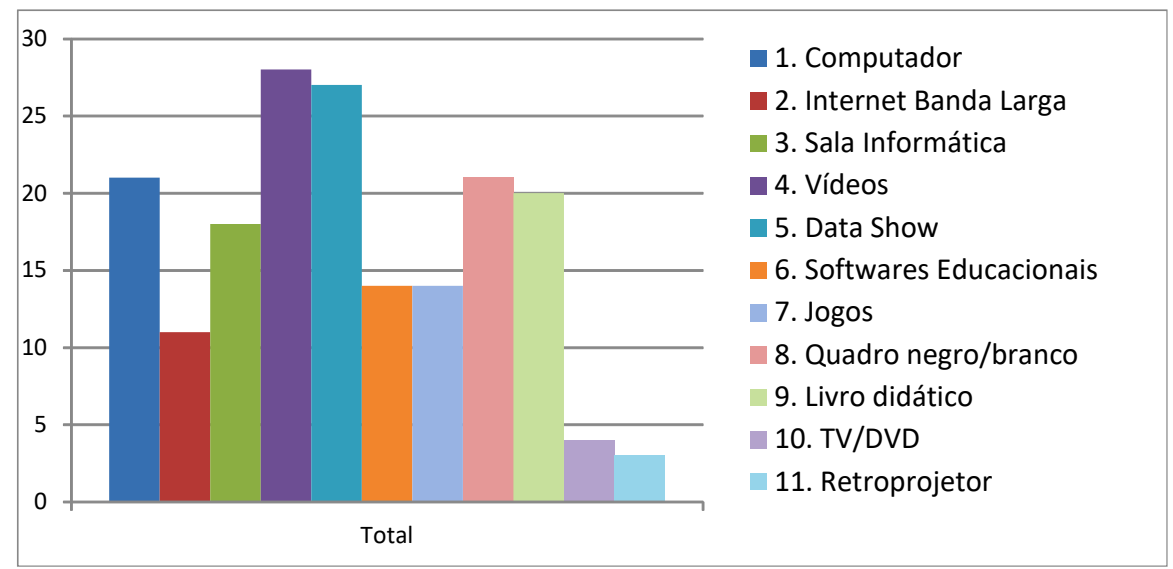

Figura 4: Incidência informada pelos alunos dos recursos utilizados que facilitam aprendizagem.

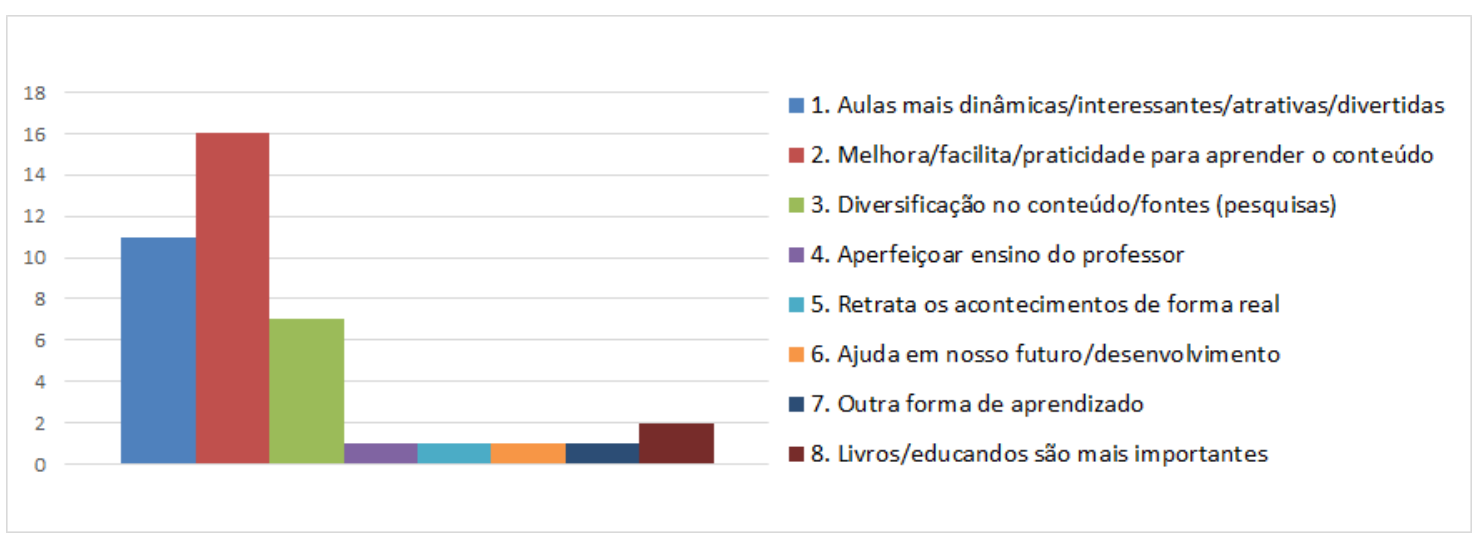

Figura 5: Frequência nas respostas abertas sobre a contribuição dos recursos tecnológicos para sua aprendizagem.

A abordagem de vídeo em sala de aula é bem discutida por Morán (1995), onde é descrito formas de uso justificando em quais momentos não se deve utilizar e como trabalhar o antes/durante/depois da exibição. Silva (2011) faz uma minuciosa exposição sobre políticas públicas a respeito do uso e como planejar o vídeo em sala, e em Ferreira e Santos (2014) também se discute melhores práticas e, a partir de um questionário, se avalia o impacto e limitações para utilização de vídeo em sala. De acordo com os estudos citados, a boa prática pode resultar em uma melhora na dinâmica da aula com maior interação dos alunos, fato constatado pelo questionário aplicado neste trabalho.

Ao descrever o que entendiam por ciência, as maiores incidências abordam temas como "abrange diversos conhecimentos/áreas" (10 vezes), "estuda o 
homem/natureza/vida" (9 vezes) e "conhecimentos comprovados por testes" (5 vezes). Lakatos (2003) e Ferrari (1974) descrevem a ciência como um conjunto de suposições organizadas de maneira lógica, correlacionadas e racionais que podem ser submetidas a verificação podendo sistematizar vários conhecimentos. Esta definição, essencialmente, corresponde com o entendimento dos alunos.

Ao expor suas definições sobre tecnologia, as principais ocorrências citam "facilitar a vida/em prol da sociedade/evolução do homem" (19 vezes) e "inovações/aprendizado/pesquisa" (10 vezes). Veraszto et al (2009) procuraram definir o significado de tecnologia a partir de uma revisão bibliográfica sistemática e, que segundo os autores:

Poderíamos dizer que a tecnologia abrange um conjunto organizado e sistematizado de diferentes conhecimentos, científicos, empíricos e intuitivos. Sendo assim, possibilita a reconstrução constante do espaço das relações humanas. (p.79)

Dessa forma, os alunos possuem um bom entendimento a respeito do que é e para o quê serve a tecnologia.

Por outro lado sobre o que são tecnologias da informação e comunicação, as respostas foram dispersas. As maiores incidências descrevem:

1. Não saber definir TIC ("não sei” - 12 vezes).

2. Que são "Meios (tecnológicos) de Comunicação" ou como "forma/meio de nos manter informados/globalização das informações" com sete vezes cada.

As TICs podem ser definidas, de maneira sucinta, como um conjunto de soluções fornecidas por recursos das telecomunicações e computação que visam a disseminação compartilhamento e armazenamento das informações (Dowbor, 2001; Souza, 2006; Pimenta, 2016). O pouco entendimento do que são TICs pode dificultar o alcance das ferramentas disponíveis para conduzir um aprendizado mais eficiente.

\section{Considerações Finais}

Foi realizada uma pesquisa com quarenta alunos de quatro escolas públicas estaduais de Pernambuco visando retratar suas percepções em relação ao uso das Tecnologias de Informação e comunicação (e demais recursos tecnológicos) - se existem, se estão sendo utilizados durante as aulas e se são abordados pelos professores.

Os resultados apontaram para relação entre os indicadores de "computador" "vídeo" e "data show" nas aulas. Esse resultado sugere que, nas escolas pesquisadas, há potencial para utilização de TICs, principalmente envolvendo vídeos. Verificou-se que há um grande acesso à Internet para "fins de comunicação (e-mail, skype e whatsapp)" por parte dos alunos. A utilização de recursos podem, na visão dos alunos, facilitar o aprendizado, fornecendo uma "praticidade para aprender o conteúdo".

A forma com que os alunos procuraram definir "ciência" e "tecnologia" demonstram um bom entendimento a respeito dos temas, apontando as melhores opções de respostas para os conceitos abordados. O intuito desta análise é realizar ações integradoras (paletras para alunos e aperfeiçoamento de professores) baseadas nas 
percepções indicadas. Dessa forma será possível trilhar um caminho ideal para o desenvolvmento das atividades posteriores.

Uma boa perspectiva se dá na utilização de dispositivos para intervenções psicopedagógicas. Como mostrado nos trabalhos de Coberlline et al (2016) e Müller e Corbellini (2016), é possível empregar TICs para proporcionar uma melhora no aprendizagem despertando um maior interesse no conteúdo abordado. De tal forma que, como mostrado nos resultados apresentados neste artigo, os alunos já utilizam ferramentas - acesso à Internet para comunicação e vídeos em aula - e acreditam que empregar recursos tecnológicos tornam as aulas mais "dinâmicas/interessantes/atrativas/divertidas". Apesar dos alunos desconhecerem as reais definições de TICs, seu objetivo é entendido.

O uso de recursos tecnológicos no processo da aprendizagem se torna cada vez mais imprescindível para atual sociedade. Portanto, o professor como detentor do conhecimento total se encerra e passa a ser um orientador de aprendizagem, socializador de saberes e formador de um pensamento crítico (Pinto e Silva, 2016; Valguima e Odakura, 2016). De tal maneira que é necessário compreender o nível de envolvimento dos professores com as TICs para auxiliar na orientação da utilização eficiente em sala de aula.

As relações entre ciência, tecnologia e TICs, se encontram na inovação tecnológica para desenvolvimento de métodos mais eficazes para ensino $\mathrm{e}$ aprendizagem. Estimular o uso e o desenvolvimento de TICs pode implicar no aumento de ferramentas mais intuitivas tanto para alunos quando para professores. A qualidade de experiência (QoE) dos usuários pode avaliar a utilização das TICs. Segundo Schimuneck et al (2014) "A Qualidade de Experiência (QoE) é uma métrica que representa o desempenho de um serviço do ponto de vista dos usuários, enfatizando a perspectiva pessoal de satisfação". Na perspectiva da informática/telecomunicações essa percepção se baseia na qualidade de serviço (QoS) e em como a aplicação multimídia é percebida pelo usuário (Valenzuela et al, 2012).

A expectativa é que, com trabalhos futuros, seja possível retratar o nível de preparo dessas escolas quanto aos aspectos de utilização às TICs relacionando a infraestrutura física disponível com a utilização consciente dos recursos. Nesse sentido, serão observados os obstáculos enfrentados pelos professores e alunos ao usar os recursos disponíveis para apontar possíveis soluções.

\section{Referências}

Bardin, L. (2011). Análise de Conteúdo. São Paulo: Edições 70.

Barvinski1, C. A. e Odakura, V. V. V. A. (2012). Desafios educacionais para o Século XXI e o papel da Informática na Educação (p. 80). In Workshop de Desafios da Computação Aplicada à Educação (desafie). Dourados, MS. Disponível em: http://www.br-ie.org/pub/index.php/desafie/article/view/2778/2431. Acessado em 13 Junho de 2017.

Corbellini, S., Real, L. C. e Silveira, N. (2016). Intervenções Psicopedagógicas e Tecnologias Digitais na Contemporaneidade (p. 1394). In Anais dos Workshops do V 
VI Congresso Brasileiro de Informática na Educação (CBIE 2017)

Anais do XXIII Workshop de Informática na Escola (WIE 2017)

Congresso Brasileiro de Informática na Educação (CBIE 2016). doi: 10.5753/cbie.wcbie.2016.1394. Disponível em: http://brie.org/pub/index.php/wcbie/article/view/7065/4939. Acessado em 19 Junho de 2017.

Ferrari, A. T. (1974) Metodologia da ciência. Rio de Janeiro: Kennedy.

Ferreira, R. D. S. e Santos, J. H. V. (2014). O uso do vídeo em sala de aula. Scientia Plena, $10(4$ (B)). Disponível em: http://scientiaplena.org.br/sp/article/view/1942. Acessado em 25 Junho de 2017.

Lakatos, E. M., e Marconi, M. de A. (2003). Fundamentos de metodologia científica. São Paulo: Atlas.

Lima, C. B. F., e Castro, J. A. F. (2016). Análise de uma Proposta Metodológica para Uso das Tecnologias Digitais no Ambiente Escolar (p. 427). In Anais dos Workshops do $V$ Congresso Brasileiro de Informática na Educação (CBIE 2016). doi:10.5753/cbie.wie.2016.427.

Massoni, N. T. (2005). Epistemologia do século XX, 3(16). Porto Alegre: UFRGS, Instituto de Física, Programa da Pós-Graduação em Ensino de Física. Disponível em: http://www.if.ufrgs.br/tapf/v16n3_Massoni.pdf. Acessado em: 22 de Junho de 2017.

Morán, J. M. (1995). O vídeo na sala de aula. Comunicação \& Educação, (2), 27-35. Disponível em: http://www.revistas.usp.br/comueduc/article/view/36131/38851. Acessado em 14 Junho de 2017.

Muller, G. C. e Corbellini, S. (2016). Tecnologias: instrumentos de intervenção psicopedagógica em dificuldade de aprendizagem (p. 1453). In Anais dos Workshops do $V$ Congresso Brasileiro de Informática na Educação (CBIE 2016). doi:10.5753/cbie.wcbie.2016.1453. Disponível em: http://brie.org/pub/index.php/wcbie/article/view/7072/4946. Acessado em 16 Junho de 2017.

Schimuneck, M. A. K., Machado, S. R. e Marotta, C. B. B. (2014). Análise da qualidade de experiência em redes sem fio. XX Seminário de Iniciação Científica. Disponível em: $\quad$ http://online.unisc.br/acadnet/anais/index.php/semic/article/view/12386. Acessado em 20 Junho de 2017.

Silva. A. M. (2011). O vídeo como recurso didático no ensino de matemática (Dissertação). Universidade Federal de Goiás, Goiânia - GO. Disponível em: http://repositorio.bc.ufg.br/tede/handle/tde/551. Acessado em 17 Junho de 2017.

Souza, R. R. R. (2006). (p. 1224). O Alcance das TICS na prática pedagógica.

In Anais do VI Congresso Nacional de educação da PUCOR. Disponível em: http://www.pucpr.br/eventos/educere/educere2006/anaisEvento/docs/CI-116-TC.pdf. Acessado em 17 Junho de 2017.

Pimenta, M. C. (2016). Tecnologia da Informação e Comunicação: a prática pedagógica do IFNMG/Campus Montes Claros. UFVJM. Disponível em: http://acervo.ufvjm.edu.br/jspui/handle/1/1170. Acessado em: 14 de Junho de 2017.

Valenzuela, M. G. et al. (2012). Mecanismo de Seleção Baseado em QoE para Redes Heterogêneas com Tráfego Multimídia. In Anais do XXXII Congresso da Sociedade Brasileira de Computação (CSBC). Centro Politécnico da Universidade Federal do Paraná. Disponível em: http://www.imago.ufpr.br/csbc2012/anais_csbc/eventos/semish/artigos/SEMISH\%20 
VI Congresso Brasileiro de Informática na Educação (CBIE 2017)

Anais do XXIII Workshop de Informática na Escola (WIE 2017)

$\%$ 20Mecanismo\%20de\%20Selecao\%20Baseado\%20em\%20QoE\%20para\%20Redes $\% 20$ Heterogeneas\%20com\%20Trafego\%20Multimidia.pdf. Acessado em: 16 de Junho de 2017.

Veraszto, E. V. et al. (2017). Tecnologia: buscando uma definição para o conceito.
Prisma.
com,
(7).
Disponível
em:

http://pentaho.letras.up.pt/ojs/index.php/prismacom/article/view/2078. Acesado em: 19 de Junho de 2017. 